\title{
Two-Degree-of-Freedom Active Vibration Control of a Prototyped "Smart" Rotor
}

\author{
Jan-Willem van Wingerden, Anton Hulskamp, Thanasis Barlas, Ivo Houtzager, Harald Bersee, Gijs van Kuik, and
} Michel Verhaegen

\begin{abstract}
This paper studies the load reduction potential of a prototyped "smart" rotor. This is, a rotor where the blades are equipped with a number of control devices that locally change the lift profile on the blade, combined with appropriate sensors and controllers. Experimental models, using dedicated system identification techniques, are developed of a scaled rotating two-bladed "smart" rotor of which each blade is equipped with trailing-edge flaps and strain sensors. A feedback controller based on $\mathcal{H}_{\infty}$-loop shaping combined with a fixed-structure feedforward control are designed that minimizes the root bending moment in the flapping direction of the two blades. We evaluated the performance using a number of different realistic load scenarios. We show that with appropriate control techniques the variance of the load signals can be reduced up to $90 \%$.
\end{abstract}

Index Terms-Fixed-structure feedforward, $\mathcal{H}_{\infty}$-control, load alleviation, trailing-edge flaps, wind energy.

\section{INTRODUCTION}

$\mathbf{T}$ HERE have been two main operation concepts to keep the loads on wind turbines (e.g., fatigue loads, power variations) within acceptable limits and to optimize the energy yield. The concept widely used from the seventies until the nineties of the previous century was the "Danish concept" [1]. Such turbines combine constant rotor speed with stall of the flow around the rotor blades at high wind speeds and are stable by design; increasing wind speeds automatically induce increasing drag forces that limit the produced power (this concept is also referred to as: stall control). In that period, all other control options were considered too complex and also the technology for variable speed control was not mature enough. Due to the development of dedicated converters, regulation concerning maximum allowable sound emissions and grid requirements, the most recent large wind turbines run at variable rotational speed, combined with the adjustment of the collective pitch angle of the blades [2]. This full-span collective pitch control is widely

Manuscript received December 14, 2009; revised March 26, 2010; accepted May 07, 2010. Manuscript received in final form May 26, 2010. Recommended by Associate Editor F. A. Cuzzola.

J. W. van Wingerden, I. Houtzager, and M. Verhaegen are with the Delft Center for System and Control, Faculty of Mechanical, Maritime, and Materials Engineering, Delft University of Technology, Delft $2628 \mathrm{CD}$, The Netherlands (j.w.vanwingerden@tudelft.nl; i.houtzager@tudelft.nl; m.verhaegen@tudelft.nl).

T. Barlas and G. A. M. van Kuik are with the Wind Energy Group, Aerospace Engineering, Delft University of Technology, Delft 2629 HS, The Netherlands (a.barlas@tudelft.nl; g.a.m.vankuik@tudelft.nl).

A. W. Hulskamp and H. E. N. Bersee are with the Design and Production of Composite Structures (DPCS), Aerospace Engineering, Delft University of Technology, Delft 2629 HS, The Netherlands (a.w.hulskamp@tudelft.nl; h.e.n. bersee@tudelft.nl).

Digital Object Identifier 10.1109/TCST.2010.2051810 accepted in the wind energy community, but can only handle slow wind changes that affect the entire rotor. Because of the increasing rotor size it is necessary to react to the distributed nature of turbulence in a more appropriate way, such as activating the blades separately and/or using local actuators at several radial distances spanwise along each blade. This first item is dealt with by individual pitch control (IPC) [3]-[6], motivated by the helicopter industry [7]-[10], which is the latest development in the wind turbine industry to further minimize the loads. With this concept each blade is pitched individually to suppress the periodic loads caused by tower shadow, wind shear, rotational sampling, yaw misalignment, etc. However, the performance of the IPC method is restricted by the limited bandwidth of and wear in the pitch actuators and because they only affect the load on the whole blade.

One can say that compared to the stall turbines, full-span collective pitch control is a step forward; the control of the blade pitch angle has not only led to power regulation, but also to a significantly lighter blade construction due to the lower load spectrum and a lighter gear box due to shaved torque peaks. With the introduction of IPC the loads can be reduced considerably leading to even lighter or larger turbines. However, due to the increasing size of wind turbines it is necessary to look ahead to control concepts which can impose a force profile matching the distributed nature of turbulence in order to reduce the loads and to guarantee an economic lifetime of 20 years for the new generation of large scale offshore wind turbines (diameter over 150 $\mathrm{m})$. So, for the next generation of wind turbines we must look for novel control concepts.

There are a large number of concepts for the next generation of wind turbines. For all of these ideas, the most important design drivers are the loads (both extreme loads and fatigue loads). One advanced operation concept is to use a number of actuators that locally change the force profile on the wind turbine blade to cope with the spatial distributed nature of turbulence. This, in combination with sensors that measure the loads and a controller that manipulates the measured signals and generates an appropriate actuation signal, is defined as the "smart" rotor concept.

The "smart" rotor concept is borrowed from the helicopter industry, where active devices like, e.g., trailing-edge flaps [11], [12], or micro-electro-mechanical translational tabs (MEM-tabs) [13] are proposed to reduce the loads. Both of these concepts manipulate the boundary layer of the flow to change the aerodynamic forces and consequently control the loads. Trailing-edge flaps are considered as the most promising idea for the "smart" rotor concept [14]. In [16] and [17], a more detailed overview is given about these developments as well as some more exotic concepts. 
The main goal of the "smart" rotor is to reduce the fatigue loads to increase the lifetime of the wind turbine. However, when the lifetime constraint is met, the concept provides the turbine designer with an increased design envelope which can be used to, for instance, mount the rotor down wind-eliminating tower clearance issues-allowing for lighter components or increasing the mean loading on the blades which increases the power conversion. Recently some research on this topic has been performed in the wind industry where trailing-edge flaps [18]-[22], and MEMtabs [23]-[25] have been used for load alleviation. The first step in the design of this new control concept was a feasibility study. This study was performed by [20], where they showed the feasibility of a trailing-edge flap applied on an airfoil (2-D study). In [26] the feasibility of the "smart" rotor concept on a rotating blade was demonstrated. The first proof of concept study was reported by [22]; a rigid cross-section with a trailing edge flap was used to validate the 2-D aerodynamic model in [21].

In [27], a 3-D experiment is presented that also takes into account the blade aeroelastic effects and a traditional single-input single-output (SISO) feedback controller, thus proving the concept of a "smart" rotor blade. This experimental work is significantly different from the work done in [22]; which used 2-D models without aeroelastics or a feedback controller.

Currently, the control methodologies used in the wind industry are mainly based on single-input single-output (SISO) gain-scheduled proportional integral differential (PID) regulators [28]. However, in the academic environments several articles are available that discuss optimal control [29]-[32], fuzzy logic control [33], model predictive control [34], [35], robust control [36], [37], and recently linear parameter-varying (LPV) control [37]-[44] of wind turbines. In general, the industry is reluctant to use these novel methodologies due to their relative complexity and the expected small performance improvement. However, with the more advanced operation concepts, such as the "smart" rotor concept, the number of control variables is increasing and it becomes necessary to use multiple-input multiple-output (MIMO) model-based control algorithms.

The main contribution of this paper is threefold. First, we bring the feasibility studies performed so far to a higher level by looking at a flexible two-bladed rotating "smart" rotor. Second, we show that by using experimental modeling, where we developed dedicated system identification tools, we circumvent the extensive modeling and parameter fitting of the aeroelastics. Third, we show the potential and the need for modern control techniques for the "smart" rotor by enabling both advanced feedback and feedforward control.

The outline of this paper is as follows. In Section II, we briefly describe the design and layout of our experimental "smart" rotor. In Section III, we introduce a dedicated system identification technique which is used to obtain models for advanced control techniques. Here the challenge is that we have to deal with dominant periodic loads (multiples of the rotational speed) and we propose a solution to solve this. In Section IV, we discuss advanced controller design. We will use the four-block $\mathcal{H}_{\infty}$ controller synthesis for disturbance rejection and since we have dominant periodic loads we will develop a dedicated fixed-structure feedforward scheme. In Section V, we present the results of both the feedback and

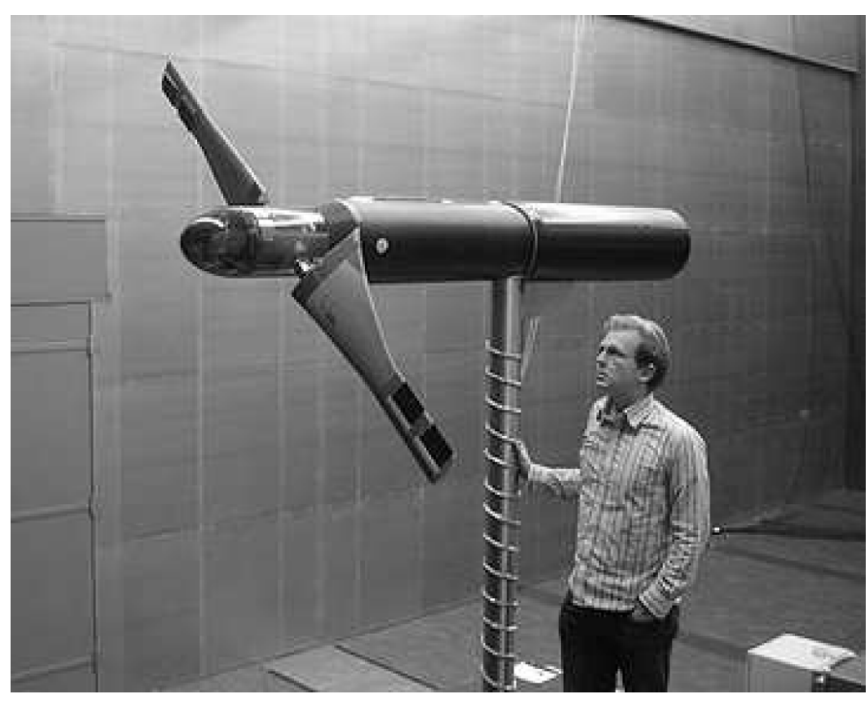

Fig. 1. Photo of the "smart" two-bladed turbine with the trailing-edge flaps The person standing next to it illustrates the size of the system.

feedforward scheme and the combination thereof. We will do this for nominal operation and the turbine standing in yaw (misalignment of the rotor). We end this paper with a number of conclusions and recommendations.

\section{DESCRIPTION OF THE EXPERIMENT}

In this section, we present the experimental setup used to show the feasibility of the "smart" rotor concept with the advanced control. The "smart" rotor that we use for our experimental validation is a rotating, two-bladed rotor equipped with trailing-edge flaps. An indication of the size and the layout of the turbine can be found in Fig. 1. Each blade is equipped with two distinct trailing-edge flaps to enable its use for future research. Since, for this study the main focus is to suppress the bending behavior of the two individual blades, the two actuators on one blade are used together as one actuator by applying the same control signal. For the same reason two strain sensors are applied in the root located on the central axis and at the leading edge of the blade, respectively. The experimental setup mainly consists of the following components: wind tunnel, blade, actuators and sensors, and real-time environment. Every element of the experimental setup will be described in this section.

\section{A. Wind Tunnel}

The experiments are conducted in the Delft University of Technology's Open Jet Facility (OJF) wind tunnel. This tunnel has a closed circuit. The air is blown into the section trough an octagonal nozzle, creating a steady jet with an effective diameter of $3 \mathrm{~m}$. There are little or no wall effects because the section is $6 \mathrm{~m}$ wide and $6.5 \mathrm{~m}$ high; much larger than the jet. The air is collected at the back of the test section, cooled and fed back into the tunnel's fan, which is powered by a $500 \mathrm{~kW}$ motor. Before the air is blown through the nozzle back in to the test section again, it enters a settling chamber with several rows of wire meshes to reduce the turbulence levels. The maximum attainable air speed is $35 \mathrm{~m} / \mathrm{s}$. 
TABLE I

SCALING PARAMETERS OF THE "SMART" ROTOR PARAMETER

\begin{tabular}{l|ll} 
& $\begin{array}{l}\text { UpWind 5MW } \\
\text { reference turbine }\end{array}$ & Scaled rotor \\
\hline Rated wind speed $(\mathrm{m} / \mathrm{s})$ & 11.4 & 7 \\
Tip speed ratio $(-)$ & 7 & 4 \\
$\begin{array}{l}\text { Rated rotational speed (rpm) } \\
\text { First mode }(\mathrm{Hz})\end{array}$ & 12 & 370 \\
(flapwise bending) & 0.69 & 24 \\
\hline
\end{tabular}

\section{B. Blade Design}

The blade was dynamically scaled such that the ratio between the rotational speed and the blade's first flapwise eigenmode was the same as with the reference design, the UpWind $5 \mathrm{MW}$ wind turbine. This is important because many of the disturbances are related to the rotation of the turbine, such as wind shear, tower shadow and yaw misalignment. These result in load fluctuations with mainly $1 \mathrm{P}, 2 \mathrm{P}$, and $3 \mathrm{P}$ frequencies at the blade's reference frame. The blade's first flapwise mode is close to the $3 \mathrm{P}$ frequency. By designing the blade such that the flapping frequency relates to the rotational frequency of the turbine, a realistic mix of rotationally induced disturbances and excited blade dynamics is obtained. See Table I for the scaling parameters. The blade's structural design consists of a glass-epoxy laminate, wrapped around a solid foam core, which was infused using vacuum assisted resin transfer moulding (VARTM) in a double rigid mould. The blade is aerodynamically designed using a blade element momentum (BEM) optimizer.

\section{Actuators and Sensors}

The most challenging part in the design of the "smart" rotor is the design of the actuator. In this paper, we design a specific actuator for the scaled two-bladed wind turbine model. The scalability is questionable with the actuator we propose; however, this is outside the scope of this research. In [14], it is shown that the most effective control device is a trailing-edge flap. Consequently, every blade was equipped with two trailing-edge flaps in the outboard part of the blade, which is where the largest aerodynamic leverage can be obtained. The flaps have a width of $10.5 \mathrm{~cm}$ in the span direction and cover half the chord length $(6.2$ $\mathrm{cm})$. The size is chosen in such a way that sufficient load reduction can be obtained for the different load cases [14]. The active part of the trailing-edge flaps consist of Thunder ${ }^{\text {tm }}$ TH-6R actuators [45]. These are piezo-electric based benders which can deflect several millimeters under the application of a maximum voltage from -450 to $900 \mathrm{~V}$. However, for safety reasons we limit the maximum voltage from -400 to $400 \mathrm{~V}$. The actual deflection also depends on the structure around the bender and the aerodynamic loading. The actuators are shaped with soft foam to give them an aerodynamic shape. The foam is covered with a skin to provide a smooth surface. The actuators are attached to the blade through a bracket that is mounted on the spar. In Fig. 2 the actuators in the tip of the blade are displayed.

For control purposes, the blade is equipped with sensors which measure the dynamic behavior of the blade. Because the final goal for this rotating "smart" rotor is to reduce the fatigue loads, two macro fiber composite (MFC) patches are adhered to the root to measure the high strains associated with the first flapwise bending

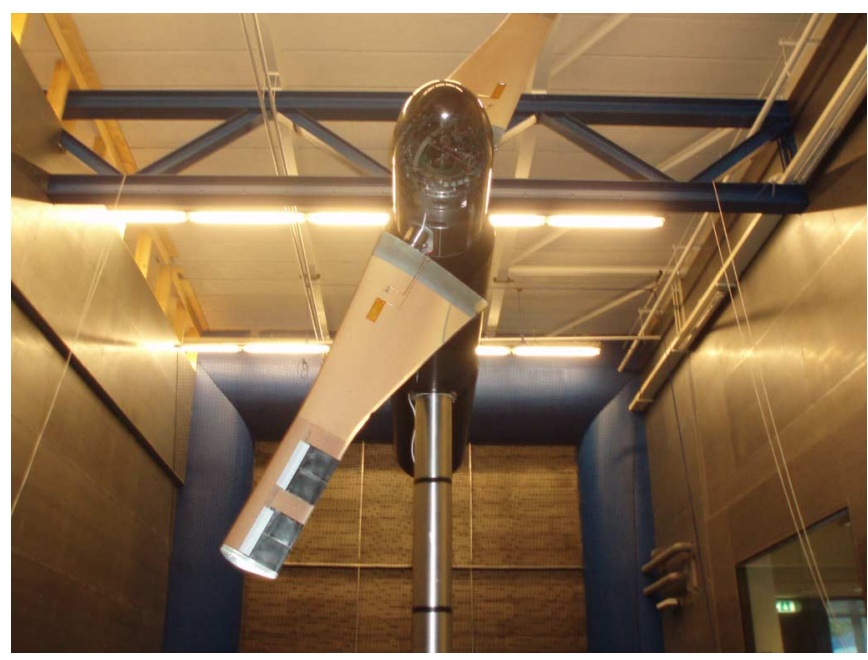

Fig. 2. Photo of the "smart" blade equipped with actuators and sensors.

mode. One MFC is placed on the pitch axis to measure the flapwise loads while the second MFC is placed near the leading edge of the blade to also measure the lead-lag loads, the objective for future experiments. The first MFC signal on both blades is used as input for the feedback controller. The main advantage of an MFC is that no amplification is required to have a good signal-to-noise ratio. However, with the MFC it is not possible to do static measurements due to the capacitance behavior of the MFC. This high pass behavior is desirable for this experiment, as we want to control the dynamic behavior of the system, rather than the static deformations. However, these static deformations may be taken into account if different sensors (e.g., strain gauges or fiber optics) are used to measure the static strain. In Fig. 2 the strain sensors can be found in the root of the blade.

\section{Real-Time Environment}

The "smart" rotor described so far is not 'smart' when there is no control enabled. This controller intelligence and data acquisition capability are added with the inclusion of a dSPACE ${ }^{\text {tm }}$ [46] chip. The controller and data acquisition scheme are fully developed in the MATLAB ${ }^{\mathrm{tm}}$ [47] and Simulink ${ }^{\mathrm{tm}}$ [48] environment and then compiled to the dSPACE ${ }^{\text {tm }}$ chip. On a separate computer all the signals are monitored using Control Desk ${ }^{\mathrm{tm}}$ [46] and the control parameters can be adjusted real-time in the same environment.

\section{EXPERIMENTAL MODELING}

The increasing number of control variables in modern wind turbines will necessitate model based controller design. In this section we first motivate that system identification is a necessary building block to come to such a controller. Second, we introduce a dedicated subspace identification algorithm to obtain the dynamics between the inputs and outputs. Finally, we present the identification results obtained on the experimental setup.

\section{A. Why System Identification?}

A model for modern model based controller design is a mathematical model normally governed by (preferably linear) differential equations. For controller synthesis this model should only contain the relevant dynamics between the input, and the output 
and should be accurate over the bandwidth of the controller. These requirements are based on implementation and conservatism issues. The two methods for obtaining a model and their application to the wind industry are listed as follows.

- First Principles (FP) Modeling: In FP modeling, the laws of physics are used to develop mathematical models. The main advantage of FP modeling is that you can have a model before the actual system is built and consequently the model can be used for system design and optimization. However, these FP models are not tailored for control requirements because they typically contain irrelevant dynamics and are nonlinear.

- Experimental Modeling (System Identification): In experimental modeling, also referred to as system identification, actual input and output data of the system is used to obtain a mathematical description of the system. Because this approach uses actual input and output data it will only model the dynamics present in the data.

Common practice is to use a mix of the two modeling approaches. This approach can be summarized as follows: develop an FP model for general design of the system and to synthesis a baseline controller. When implemented on the real system, the model used for controller synthesis should be refined by using system identification to obtain more accurate models over the bandwidth to obtain less conservative controllers.

The analytical modeling which has been performed in the design stage strongly depends on a large number of parameters which completely determine the dynamic behavior of the system. Most of these parameters can be roughly estimated or calculated. Still, a large amount of uncertainty is present; this makes it hard to design a stable feedback controller based on such models. In this section we use experimental modeling to build a linear dynamic model. A subspace identification method will be used because of the numerical simplicity and the potential to work with MIMO systems [49], [50]. The MIMO approach is necessary since there is a high degree of coupling between the different actuators and sensors.

\section{B. Algorithm}

The system of interest, the two-bladed "smart" rotor significantly experiences periodic loads which are multiples of the rotational speed, we refer to this as $1 \mathrm{P}, 2 \mathrm{P}, \ldots$ etc. For system identification these loads are disturbances which we do not want to model between the input and output of the system. In this section we present a modified identification scheme that can cope with periodic disturbances that can be expressed as a linear combination of known periodic functions. Since, we more or less know that the disturbances are sinusoids of a given frequency $(1 \mathrm{P}, 2 \mathrm{P}, \ldots)$ with unknown amplitudes and phases. The following steps are taken.

1) Problem Formulation: For the derivation of the algorithm we consider the following linear system with periodic disturbances of which we have some prior knowledge: ${ }^{1}$

$$
\begin{aligned}
x_{k+1} & =A x_{k}+B u_{k}+G d_{k}+K e_{k} \\
y_{k} & =C x_{k}+e_{k}
\end{aligned}
$$

${ }^{1}$ We disregard the direct feedthrough term for the sake of clarity of presentation. where $x_{k} \in \mathbb{R}^{n}, u_{k} \in \mathbb{R}^{r}$ and $y_{k} \in \mathbb{R}^{\ell}$, are the state, input (trailing-edge deflections in $[\mathrm{V}]$ ) and output vectors (strain measurements in $[\mathrm{V}]$ ), respectively. $e_{k} \in \mathbb{R}^{\ell}$ denotes the zero mean white innovation process. Where $d_{k} \in \mathbb{R}^{m}$ contains the basis functions needed to express the periodic disturbances. The matrices $A \in \mathbb{R}^{n \times n}, B \in \mathbb{R}^{n \times r}, C \in \mathbb{R}^{\ell \times n}, K \in \mathbb{R}^{n \times \ell}, G \in$ $\mathbb{R}^{n \times m}$, are the system, input, output, observer, and periodic noise matrices, respectively. We can rewrite (1)-(2) in the predictor form as

$$
\begin{aligned}
x_{k+1} & =\tilde{A} x_{k}+B u_{k}+G d_{k}+K y_{k} \\
y_{k} & =C x_{k}+e_{k}
\end{aligned}
$$

with

$$
\tilde{A}=A-K C \text {. }
$$

It is well-known that an invertible linear transformation of the state does not change the input-output behavior of this model structure. These transformations are given by: $T^{-1} A T, T^{-1} B, T^{-1} G, T^{-1} K$, and $C T$ with $T \in \mathbb{R}^{n \times n}$.

The identification problem can now be formulated as: given the input sequence $u_{k}$, the basis functions of the periodic disturbance signals $d_{k}$, and the output sequence $y_{k}$ over a time $k=\{1, \ldots, N\}$; find all, if they exist, the system matrices $A, B, G, K$, and $C$ up to the mentioned similarity transformation.

2) Assumptions and Notation: We define the following stacked vectors:

$$
U_{k}^{(s)}=\left[\begin{array}{llll}
u_{k}^{T}, & u_{k+1}^{T}, & \ldots, & u_{k+s-1}^{T}
\end{array}\right]^{T}
$$

and similar vectors exist for the signals $y_{k}$, and $d_{k}$, given by $Y_{k}^{(s)}$ and $D_{k}^{(s)}$, respectively. We also define the extended controllability matrix

$$
\mathcal{K}=\left[\mathcal{K}_{u}, \quad \mathcal{K}_{y}, \mathcal{K}_{d}\right]
$$

with

$$
\begin{aligned}
\mathcal{K}_{u} & =\left[\begin{array}{llll}
\tilde{A}^{p-1} B, & \tilde{A}^{p-2} B, & \ldots, & B
\end{array}\right] \\
\mathcal{K}_{y} & =\left[\begin{array}{llll}
\tilde{A}^{p-1} K, & \tilde{A}^{p-2} K, & \ldots, & K
\end{array}\right] \\
\mathcal{K}_{d} & =\left[\begin{array}{llll}
\tilde{A}^{p-1} G, & \tilde{A}^{p-2} G, & \ldots, & G
\end{array}\right]
\end{aligned}
$$

where $p$ is referred to as the past window. We also define the following Toeplitz matrices:

$$
\begin{aligned}
\tilde{H}(B) & =\left[\begin{array}{ccccc}
0 & 0 & 0 & \cdots & 0 \\
C B & 0 & 0 & \cdots & 0 \\
C \tilde{A} B & C B & 0 & \cdots & 0 \\
\vdots & \ddots & \ddots & \ddots & \\
C \tilde{A}^{f-2} B & C \tilde{A}^{f-3} B & C \tilde{A}^{f-4} B & \cdots & 0
\end{array}\right], \\
H(B) & =\left[\begin{array}{ccccc}
0 & 0 & 0 & \cdots & 0 \\
C B & D & 0 & \cdots & 0 \\
C A B & C B & 0 & \cdots & 0 \\
\vdots & \ddots & \ddots & \ddots & \\
C A^{f-2} B & C A^{f-3} B & C A^{f-4} B & \cdots & 0
\end{array}\right]
\end{aligned}
$$


where $f$ is referred to as the future window. We also define the observability matrices, $\tilde{\Gamma}$ and $\Gamma$

$$
\tilde{\Gamma}=\left[\begin{array}{c}
C \\
C \tilde{A} \\
\vdots \\
C \tilde{A}^{f-1}
\end{array}\right], \quad \Gamma=\left[\begin{array}{c}
C \\
C A \\
\vdots \\
C A^{f-1}
\end{array}\right] .
$$

Next, we briefly describe the idea of predictor-based subspace identification $\left(\mathrm{PBSID}_{\mathrm{opt}}\right)$ only now with periodic disturbances.

3) Predictors: The first objective of the predictor based subspace algorithms is to reconstruct the state up to a similarity transformation. Given the previous definitions the state $x_{k+p}$ is given by

$$
x_{k+p}=\tilde{A}^{p} x_{k}+\left[\mathcal{K}_{u}, \quad \mathcal{K}_{y}, \quad \mathcal{K}_{d}\right]\left[\begin{array}{c}
U_{k}^{(p)} \\
Y_{k}^{(p)} \\
D_{k}^{(p)}
\end{array}\right] .
$$

The key approximation in this algorithm is that we assume that $\tilde{A}^{j} \approx 0$, for all $j \geq p$. It can be shown that if the system in (3)-(4) is uniformly exponentially stable, the approximation error can be made arbitrarily small by making $p$ sufficiently large [51]. With this assumption the state $x_{k+p}$ is approximately given by 2,3

$$
x_{k+p} \approx\left[\begin{array}{lll}
\mathcal{K}_{u}, & \mathcal{K}_{y}, & \mathcal{K}_{d}
\end{array}\right]\left[\begin{array}{c}
U_{k}^{(p)} \\
Y_{k}^{(p)} \\
D_{k}^{(p)}
\end{array}\right] .
$$

In a number of other LTI subspace methods it is well known to make this step (e.g., N4SID, SSARX, and CVA). The inputoutput behavior is now approximately given by

$$
y_{k+p} \approx C\left[\mathcal{K}_{u}, \quad \mathcal{K}_{y}, \quad \mathcal{K}_{d}\right]\left[\begin{array}{c}
U_{k}^{(p)} \\
Y_{k}^{(p)} \\
D_{k}^{(p)}
\end{array}\right]+e_{k+p} .
$$

This equation is a regression problem, an estimate of the matrix $C \mathcal{K}$ can be obtained by solving a linear problem by stacking the data over the $N-p$ data points

$$
\begin{aligned}
Y & =\left[\begin{array}{lll}
y_{1+p}, & y_{2+p}, \ldots, & y_{N}
\end{array}\right] \\
Z & \left.=\left[\begin{array}{c}
U_{1}^{(p)} \\
Y_{1}^{(p)} \\
D_{1}^{(p)}
\end{array}\right],\left[\begin{array}{c}
U_{2}^{(p)} \\
Y_{2}^{(p)} \\
D_{2}^{(p)}
\end{array}\right], \ldots,\left[\begin{array}{c}
U_{N-p}^{(p)} \\
Y_{N-p}^{(p)} \\
D_{N-p}^{(p)}
\end{array}\right]\right] .
\end{aligned}
$$

With which we can formulate the following linear problem:

$$
C\left[\mathcal{K}_{u}, \min _{\mathcal{K}_{y},} \mathcal{K}_{d}\right]\left\|Y-C\left[\mathcal{K}_{u}, \quad \mathcal{K}_{y}, \quad \mathcal{K}_{d}\right] Z\right\|_{F}^{2}
$$

where we add an additional constraint that we obtain the minimal norm solution, $\left\|C\left[\mathcal{K}_{u}, \quad \mathcal{K}_{y}, \quad \mathcal{K}_{d}\right]\right\|_{F}^{2}$, since the matrix $Z$

\footnotetext{
${ }^{2}$ Remark: If the matrix $(A-K C)$ is nilpotent the assumption can be removed. Since, in that case it holds that there exists a $p$ such that $(A-K C)^{p}=0$.

${ }^{3}$ Remark: In the case that there is no noise present the observer gain will be a deadbeat observer and under the conditions stated the following will hold: $(A-K C)^{p}=0$ for $p \geq n$.
}

is likely to have no full row rank. Since periodic signals stacked in a Hankel matrix can make this matrix rank deficient and consequently additional constraints should be added to guarantee a unique solution [52].

4) Extended Observability Times Controllability Matrix: The product $\mathcal{K} Z$ that represents by definition the state sequence, $X_{p}=\left[x_{1+p}, \ldots, x_{N}\right]$, cannot be estimated directly. In the predictor-based identification algorithms $C \mathcal{K}$ is used to construct the extended observability matrix times the extended controllability matrix. This matrix is given by

$$
\begin{aligned}
\tilde{\Gamma} \mathcal{K} & =\left[\begin{array}{ccc}
C \mathcal{K}_{u} & C \mathcal{K}_{y} & C \mathcal{K}_{d} \\
C \tilde{A} \mathcal{K}_{u} & C \tilde{A} \mathcal{K}_{y} & C \tilde{A} \mathcal{K}_{d} \\
\vdots & \vdots & \\
C \tilde{A}^{f-1} \mathcal{K}_{u} & C \tilde{A}^{f-1} \mathcal{K}_{y} & C \tilde{A}^{f-1} \mathcal{K}_{d}
\end{array}\right] \\
& =\left[\begin{array}{lll}
\tilde{\Gamma} \mathcal{K}_{u} & \tilde{\Gamma} \mathcal{K}_{y} & \tilde{\Gamma} \mathcal{K}_{d}
\end{array}\right] .
\end{aligned}
$$

If we look into more detail at for instance the $\tilde{\Gamma} \mathcal{K}_{u}$ term we have for the PBSID $_{\text {opt }}$ algorithm (we assume $f=p$ )

$$
\tilde{\Gamma} \mathcal{K}_{u} \approx\left[\begin{array}{cccc}
C \tilde{A}^{p-1} B & C \tilde{A}^{p-2} B & \cdots & C B \\
0 & C \tilde{A}^{p-1} B & \cdots & C \tilde{A} B \\
\vdots & & \ddots & \\
0 & 0 & & C \tilde{A}^{p-1} B
\end{array}\right] .
$$

The zeros appear in this equation based on the approximation that $\tilde{A}^{j} \approx 0$ for all $j \geq p$. Although, in [51] it was shown that these zeros appear in the solution based on the result of an optimization problem. Observe that from the solution of the linear problem formulated in (5) we can construct the matrix $\tilde{\Gamma} \mathcal{K}_{u}$. A similar procedure can be followed for the matrices $\tilde{\Gamma} \mathcal{K}_{y}$ and $\tilde{\Gamma} \mathcal{K}_{d}$.

From the constructed matrix $\tilde{\Gamma} \mathcal{K}$ we can compute $\tilde{\Gamma} \mathcal{K} Z$, which equals by definition the extended observability times the state sequence, $\tilde{\Gamma} X_{p}$. By computing a singular value decomposition (SVD) of this estimate we can estimate the state sequence and the order of the system. We will use the following SVD:

$$
\widehat{\widetilde{K}} Z=\left[\begin{array}{ll}
\mathcal{U} & \mathcal{U}_{\perp}
\end{array}\right]\left[\begin{array}{cc}
\Sigma_{n} & 0 \\
0 & \Sigma
\end{array}\right]\left[\begin{array}{c}
\mathcal{V} \\
\mathcal{V}_{\perp}
\end{array}\right]
$$

where $\Sigma_{n}$ is the diagonal matrix containing the $n$ largest singular values and $\mathcal{V}$ is the corresponding row space. Note that we can find the largest singular values by detecting a gap between the singular values [50]. The state is now estimated by

$$
\hat{X}_{p}=\Sigma_{n} \mathcal{V} .
$$

It is well known that when the state, input, and output are known the system matrices can be estimated [50]. This briefly summarizes the modified identification algorithm. In the remainder of this section the performance of this algorithm is evaluated on the experimental setup which was described in the previous section.

\section{Identification Cycle}

The control loops in the prototyped "smart" rotor are the transfer functions between the trailing-edge flaps on the two different blades $(u)$, and the corresponding MFC sensors $(y)$. The performance of the subspace identification is evaluated by 


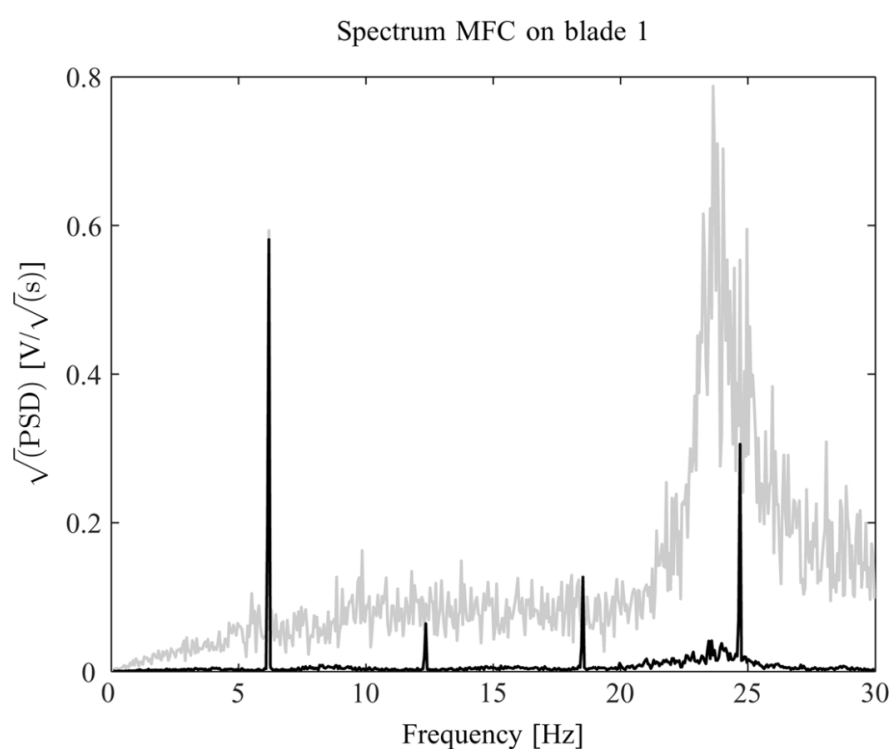

Fig. 3. Square-root of the PSD of the MFC signal on blade 1. The grey line is with GBN excitation and the black one is without excitation. In this figure we clearly see the periodic disturbances on the 1P, 2P, 3P, and 4P frequencies. For $V=7 \mathrm{~m} / \mathrm{s}$ and $\omega=370 \mathrm{r} / \mathrm{min}$.

looking at the variance-accounted-for (VAF) on a data set different from the data set used for determining the model. The VAF value is defined as

$$
\mathrm{VAF}=\max \left\{1-\frac{\operatorname{var}(y-\hat{y})}{\operatorname{var}(y)}, 0\right\} * 100 \%
$$

where $\hat{y}$ denotes the output signal obtained by simulating the identified system, $y$ is the measured signal, and $\operatorname{var}()$ denotes the variance of a quasi-stationary signal.

The subspace identification is done with a GBN signal with a bandwidth of $50 \mathrm{~Hz}$ with an amplitude of $400 \mathrm{~V}$ on the two actuators (almost maximum excitation) the data is acquired with a sampling time of $100 \mathrm{~Hz}$. In Fig. 3 we see the square-root of the power-spectral density with and without GBN excitation. With no excitation we clearly see peaks at the 1P-4P frequencies mainly due to the unbalance of the two-bladed rotor. When we excite the system we see that especially at higher frequencies we have a good signal-to-noise ratio (SNR). However, the 1P is dominantly present and that is the main reason that we introduced the identification scheme that can handle these periodic disturbances. Second, this figure also indicates the control authority of the actuators where we can conclude that the control authority at the $1 \mathrm{P}$ frequency is rather low.

Identification can be done for different operating points. We performed the identification experiments for two operating conditions: one for $7 \mathrm{~m} / \mathrm{s}$, and a rotation speed of $370 \mathrm{r} / \mathrm{min}$ and one for the $10 \mathrm{~m} / \mathrm{s}$ situation but now with $430 \mathrm{r} / \mathrm{min}$, both with a pitch angle setting of $4^{\circ}$ (pitch to feather). For the disturbance signal we take for the $1 \mathrm{P}$ until the $3 \mathrm{P}$ a set of cosines and sinusoids of the corresponding frequency, which can form together a sinusoid at that particular frequency with a certain amplitude and phase shift. We compare the modified identification scheme with the original scheme by looking at the VAF values which can be found in Table II. We clearly can see the benefits of the modified scheme. For the modified and the original scheme the Bode magnitude plots are given in Fig. 4. They are compared with their frequency domain estimate (SPA) which is also using the additional periodic basis functions.

From Fig. 4 one resonance peak can be observed which is consistently estimated; this one represents the first flapping mode. We clearly see the benefits of the modified scheme since the original PBSID opt scheme does not know what to do with the dominant periodic disturbance. Further, we can clearly see that there is a strong coupling between the different blades. That is why we will apply MIMO control in the next section. The VAF values are higher for the higher wind speed. This is caused by the fact that with similar excitation the measured signals are larger and consequently the SNR is higher, which has a direct effect on the VAF.

\section{Two-DEGREE-OF-FrEEDOM (2DOF) CONTROLlER DESIGN}

The main focus of the "smart" rotor concept is to reduce the fatigue loads on the complete rotor. For controller design it is important to know the dynamic relation between the actuators used for control, the control device, and the sensors used for control. The obtained state-space model using subspace identification describes the desired relation. In Fig. 4 the dynamic relation between the actuators and sensors is given by means of a Bode magnitude plot. In industry it is common practice to use the Bode plot between the input and output to design a feedback controller. The goal of the feedback controller is to suppress the unknown disturbances as much as possible; however, the ability to do so is limited by the requirement that the system should remain stable; a bounded input will result in a bounded output. With feedforward control you can deal with deterministic disturbances without the problem of instability. However, you need accurate knowledge of the disturbances present. We already indicated that for wind turbines a large component of the loads is caused by deterministic periodic disturbances with a frequency equal to the rotorspeed and multiples thereof but there is also a strong stochastic component present. The proposed control strategy is a 2DOF controller that combines a feedforward and feedback scheme. In this section first we will present a fixed-structure feedforward scheme with a novel data-driven tuning strategy. We combine this feedforward algorithm with a MIMO $\mathcal{H}_{\infty}$ controller to mitigate the stochastic disturbances.

\section{A. Feedforward Control}

Since we know the shapes and frequencies of the most dominant deterministic disturbances we can embed them in a feedforward signal. However, we do not know the phase and amplitude necessary to compensate for the disturbances. To do so we have to learn these properties. These properties can be linearly parameterized by defining the following fixed-structure feedforward signal:

$$
u_{k}^{(f f)}=\sum_{i=1}^{q} \theta_{i}^{s} \sin (i \omega t)+\theta_{i}^{c} \cos (i \omega t)
$$

where $t$ in $s$ is time and $\omega$ the rotational speed in $\mathrm{rad} / \mathrm{s}$. Further, we have the unknowns $\theta_{i}^{s}$ and $\theta_{i}^{c} \in \mathbb{R}^{r}$ and $q$ the number of periodic components that should be taken into account. The fixed-structure feedforward scheme is illustrated in Fig. 5. The question appears how do we select these $\theta$ 's? We use ideas 
From Flap blade $1[\mathrm{~V}]$
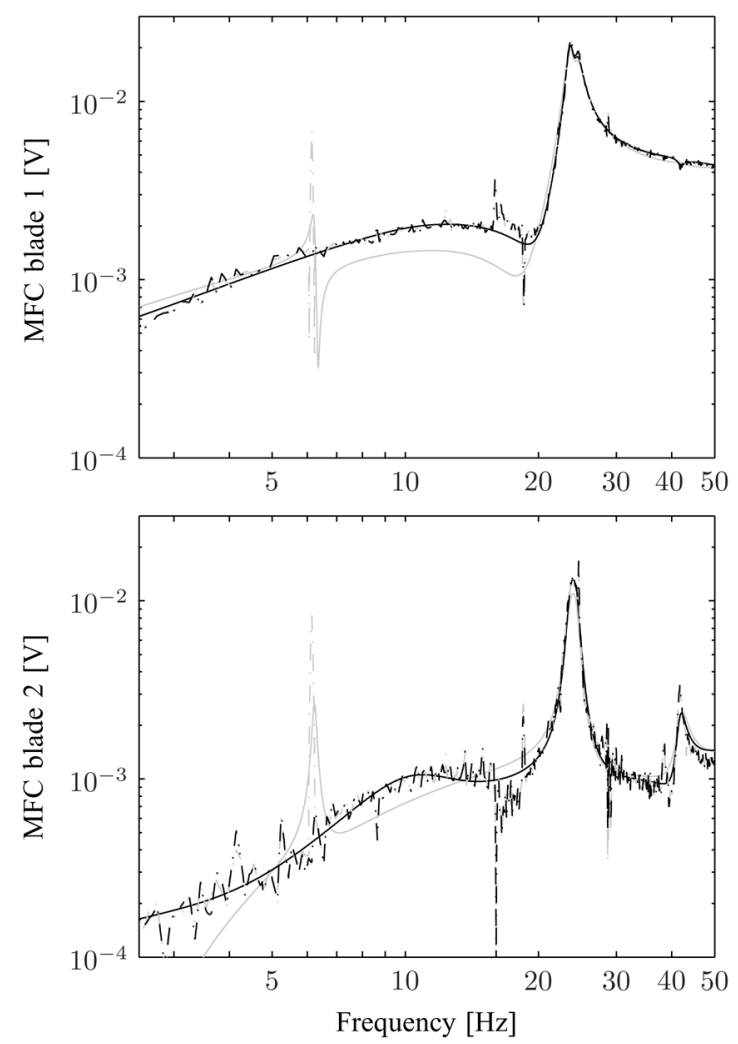

From Flap blade $2[\mathrm{~V}]$
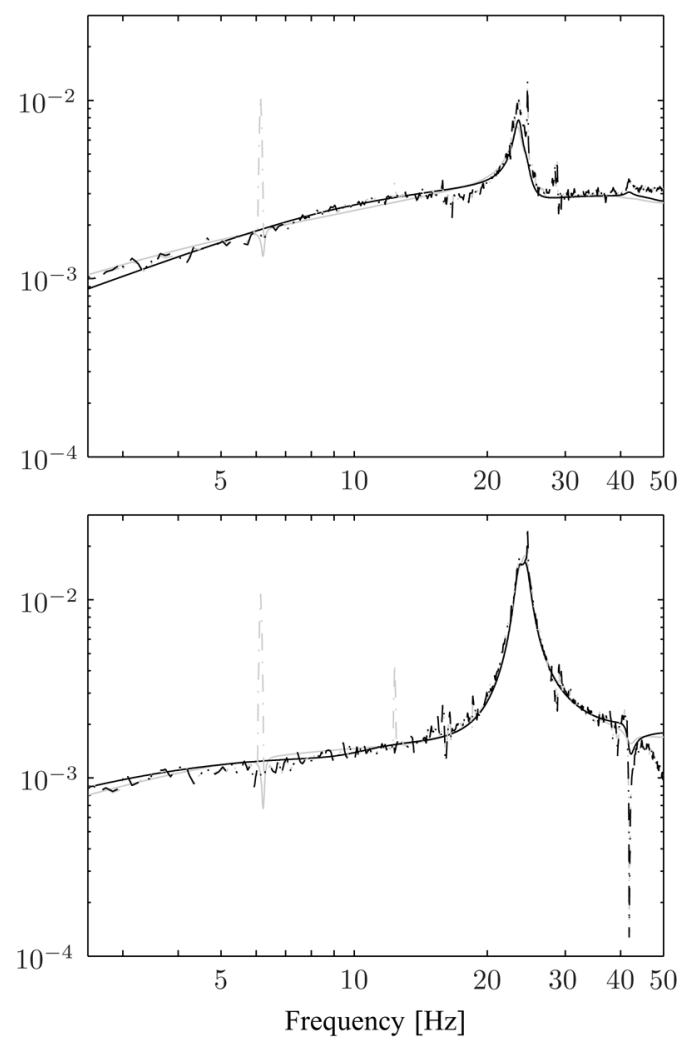

Fig. 4. Frequency versus magnitude plots of the identified eighth-order model using the modified PBSID $_{\text {opt }}$ (black line) algorithm and the original PBSID ${ }_{\text {opt }}$ algorithm (grey line). Also the corresponding frequency estimates are given with the dashed lines with corresponding color (SPA). The models are valid for $V=$ $7 \mathrm{~m} / \mathrm{s}$ and $\omega=370 \mathrm{r} / \mathrm{min}$.

TABLE II

VARIANCE ACCOUNTED FOR (VAF) FOR THE DIFFERENT IDENTIFICATION METHODS AND FOR DIFFERENT OPERATING POINTS

\begin{tabular}{|c|c|c|c|}
\hline \multicolumn{2}{|c|}{ PBSID $_{\text {opt }}$} & \multicolumn{2}{|l|}{ VAF MFC's } \\
\hline $\mathrm{V}[\mathrm{m} / \mathrm{s}]$ & RPM & blade $1[\%]$ & blade $2[\%]$ \\
\hline$\overline{7}$ & 370 & 93.6 & 87.9 \\
\hline 10 & 430 & 97.1 & 92.3 \\
\hline PB & odified & VAF MFC's & \\
\hline $\mathrm{V}[\mathrm{m} / \mathrm{s}]$ & RPM & blade $1[\%]$ & blade $2[\%]$ \\
\hline$\overline{7}$ & 370 & 97.9 & 94.8 \\
\hline 10 & 430 & 97.6 & 94.2 \\
\hline
\end{tabular}

from repetitive control to learn these parameters (see [53] and references therein). ${ }^{4}$ In the previous section, we discussed predictor-based subspace identification for LTI systems with periodic disturbances. In this section, we use the same algebra to find the optimal $\theta$ 's. A comprehensive description of the algorithm can be found in the following.

1) Data Equation With Period Definition: First we define the predictor over one prediction horizon $P$. This predictor is given by: 5

$\hat{Y}_{k+p}^{(P)}=\tilde{\Gamma} \mathcal{K}\left[\begin{array}{c}U_{k}^{(p)} \\ Y_{k}^{(p)} \\ D_{k}^{(p)}\end{array}\right]+[\tilde{H}(B), \quad \tilde{H}(K), \quad \tilde{H}(G)]\left[\begin{array}{c}U_{k+p}^{(P)} \\ \hat{Y}_{k+p}^{(P)} \\ D_{k+p}^{(P)}\end{array}\right]$

${ }^{4}$ The algorithm assumes that the rotor speed is more or less constant which is a reasonable assumption for the above rated situation. A question for future research is if the algorithm is also suitable for the below rated region.

${ }^{5}$ Note, that this is a predictor since we ignore the white noise sequence. where $P$ is the number of samples contained in one revolution. The predicted output $\hat{Y}_{k+p}^{(P)}$ is present at the left-hand side and the right-hand side of the equation. By moving this last term to the left-hand side we obtain the following equation: ${ }^{6}$

$$
\hat{Y}_{k+p}^{(P)}=\Gamma \mathcal{K}\left[\begin{array}{c}
U_{k}^{(p)} \\
Y_{k}^{(p)} \\
D_{k}^{(p)}
\end{array}\right]+[H(B), \quad H(G)]\left[\begin{array}{c}
U_{k+p}^{(P)} \\
D_{k+p}^{(P)}
\end{array}\right] .
$$

This can be interpreted as going back from the predictor formulation to the innovation formulation. We can have the same equation for one period in the future and subtract it. We define the following operator: $\delta U_{k+p}^{(P)}=U_{k+p}^{(P)}-U_{k+p-P}^{(P)}$. We obtain

$\hat{Y}_{k+p}^{(P)}=\underbrace{\left[\begin{array}{lll}\Gamma \mathcal{K}_{u}, & \Gamma \mathcal{K}_{y}, & I\end{array}\right]}_{M}\left[\begin{array}{c}\delta U_{k}^{(p)} \\ \delta Y_{k}^{(p)} \\ Y_{k+p-P}^{(P)}\end{array}\right]+H(B)\left[\delta U_{k+p}^{(P)}\right]$.

Due to the $\delta$-operator the periodic components will drop-out of the equations. This data equation will be used in the remainder of this algorithm to derive the update law of the fixed-structure feedforward scheme.

${ }^{6}$ For identification it does not make sense to make the control horizon larger than the past horizon but for control is does. 


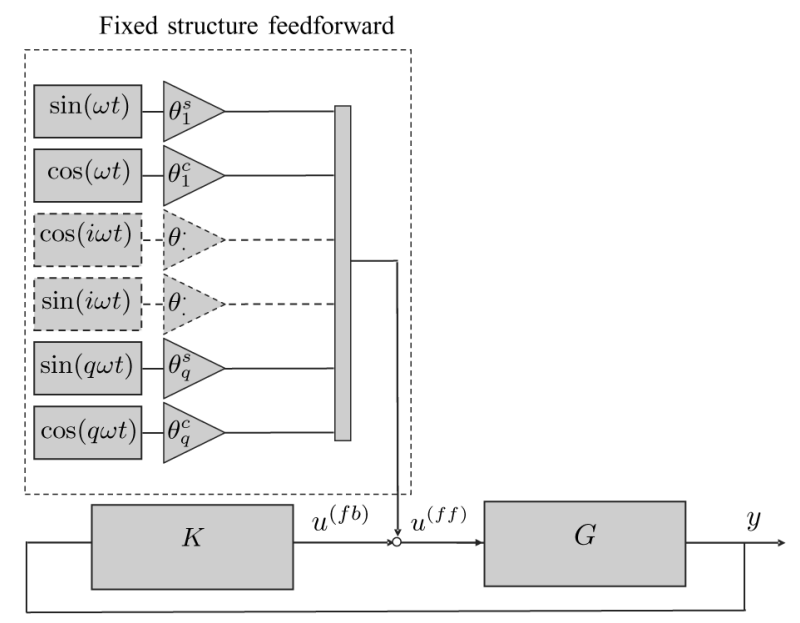

Fig. 5. Illustration of the fixed-structure feedforward scheme in combination with feedback.

2) Minimization of the Error: For vibration control we would like to minimize the future output this can be formulated in the following objective function which we would like to minimize:

$$
\min _{U_{k+p}^{(P)}}\left\|\left(\hat{Y}_{k+p}^{(P)}\right)^{T} \hat{Y}_{k+p}^{(P)}\right\|_{F}^{2}
$$

If there are no constraints added, which is possible, the analytic solution of this problem is given by

$U_{k+p}^{(P)}=U_{k+p-P}^{(P)}-\underbrace{\left(H(B)^{T} H(B)\right)^{-1} H(B)^{T} M}_{L}\left[\begin{array}{c}\delta U_{k}^{(p)} \\ \delta Y_{k}^{(p)} \\ Y_{k+p-P}^{(P)}\end{array}\right]$

where $L$ is the update gain which can be computed offline. This equation is a typical repetitive controller update law. To make this update law adaptive normally a forgetting factor is added $\beta$ and to tune the convergence an update gain is added $\alpha$. So, in general we have

$$
U_{k+p}^{(P)}=\beta U_{k+p-P}^{(P)}-\alpha L\left[\begin{array}{c}
\delta U_{k}^{(p)} \\
\delta Y_{k}^{(p)} \\
Y_{k+p-P}^{(P)}
\end{array}\right]
$$

3) Basis Functions: At the beginning of this section we already parameterized the input space of the feedforward signal. With this factorization we can rewrite $U_{k+p}^{(P)}$ as

$$
U_{k+p}^{(P)}=U^{f f} \theta_{k+p}^{(P)}
$$

where $U_{f f}$ is defined in the equation at the bottom of the page, where $t_{s}$ is the sampling time and $\otimes$ the Kronecker product. Further

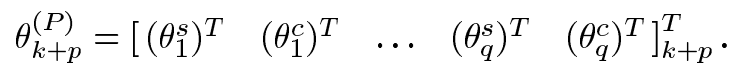

With this substitution the update scheme now reads

$$
\theta_{k+p}^{(P)}=\beta \theta_{k+p-P}^{(P)}-\alpha L^{\prime}\left[\begin{array}{c}
\delta U_{k}^{(p)} \\
\delta Y_{k}^{(p)} \\
Y_{k+p-P}^{(P)}
\end{array}\right]
$$

with $\left.L^{\prime}=\left(H(B) U^{(f f)}\right)^{T} H(B) U^{(f f)}\right)^{-1}\left(H(B) U^{(f f)}\right)^{T} M$ which can be computed offline. These $\theta$ 's can now directly be used in the feedforward configuration sketched in Fig. 5. These $\theta$ 's can be learned in a dedicated period or they can be adjusted continuously by tuning the $\alpha$ and $\beta$. The performance of the feedforward will be evaluated in Section $\mathrm{V}$ in combination with feedback control.

\section{B. Feedback Control}

Since not all the disturbances are deterministic we have to add a feedback controller. We have already mentioned that there is strong coupling between the different actuators and sensors. Although one can consider decoupling of the different feedback loops this becomes more and more difficult when there are multiple flaps active on one blade that is why we design a MIMO feedback controller. For a stable SISO non-minimum phase system, manual loop-shaping is a well known method to design stable feedback controllers based on the Bode plot of the system of interest. If we consider the input from blade one to the MFC on blade one (SISO), a smoothed proportional-derivative (PD) controller with additional inverted notch filters at the 1P-4P frequencies will make a stable feedback controller with a considerable amount of disturbance rejection at the P-frequencies. However, this controller will only give performance on one blade and we measured performance degradation on the other blade. This again motivates why we use a MIMO controller. For the controller synthesis we use the so-called four block $\mathcal{H}_{\infty}$ control synthesis setting which is illustrated in the well-known generalized plant setting in Fig. 6 (see also [54]). The $\mathcal{H}_{\infty}$ controller will minimize the $\mathcal{H}_{\infty}$ norm between $\left\{d_{1}, d_{2}\right\}$ and $\left\{z_{1}, z_{2}\right\}$ or mathematically given by

$$
\left\|\left[\begin{array}{cc}
W_{z 1} K(I-G K)^{-1} & W_{z 1}(I-K G)^{-1} \\
W_{z 2}(I-G K)^{-1} & W_{z 2}(I-G K)^{-1} G
\end{array}\right]\right\|_{\infty} .
$$

In these objective functions we can embed the manual loop shaping ideas in the weighting filters $W_{z 1}$ and $W_{z 2}$. We choose $W_{z 2}$ in such a way that we penalize the $1 \mathrm{P}-4 \mathrm{P}$ frequencies by including inverted notches at these frequencies and we also add

$$
U^{f f}=\ldots\left[\begin{array}{ccccc}
\sin \left(\omega t_{s}\right) & \cos \left(\omega t_{s}\right) & \ldots & \sin \left(q \omega t_{s}\right) & \cos \left(q \omega t_{s}\right) \\
\vdots & \vdots & \ldots & \vdots & \vdots \\
\sin \left(\omega P t_{s}\right) & \cos \left(\omega P t_{s}\right) & \ldots & \sin \left(q \omega P t_{s}\right) & \cos \left(q \omega P t_{s}\right)
\end{array}\right] \otimes I_{r}
$$




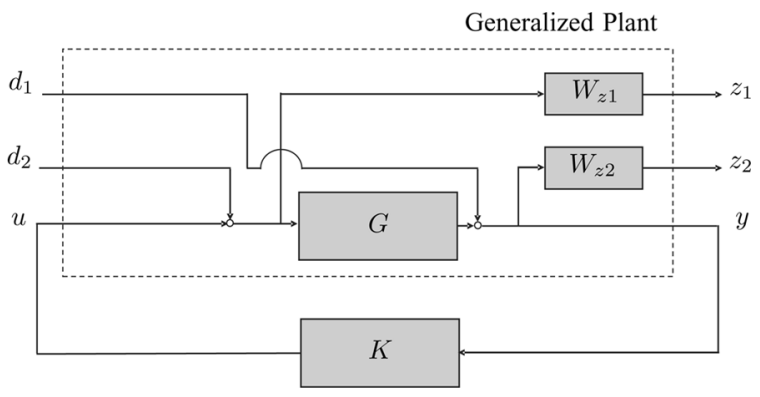

Fig. 6. Illustration of the generalized plant used to synthesize the four block $\mathcal{H}_{\infty}$ controller.

Spectrum MFC on blade 1
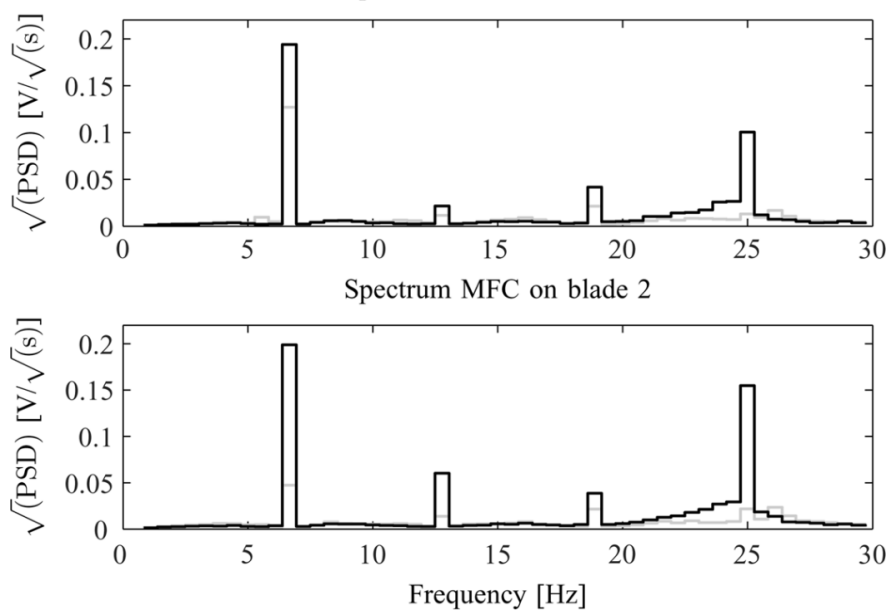

Fig. 7. Square-root of the PSD of the MFC signal on blade one and two. The grey line is with $\mathcal{H}_{\infty}$ feedback control and the black one is without control. For $V=7 \mathrm{~m} / \mathrm{s}$ and $\omega=370 \mathrm{r} / \mathrm{min}$.
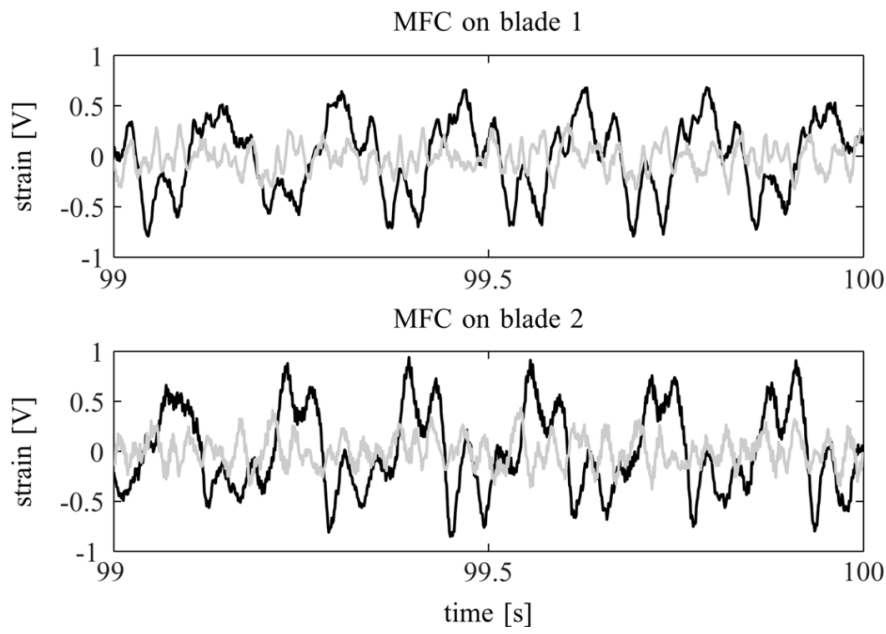

Fig. 8. Time domain performance of the controller evaluated on the MFC signal on blade one and two. The grey line is with $\mathcal{H}_{\infty}$ feedback and fixed-structure feedforward control and the black one is without any control. For $V=7 \mathrm{~m} / \mathrm{s}$ and $\omega=370 \mathrm{r} / \mathrm{min}$.

a notch at the first flapping mode to obtain damping enhancement. We choose $W_{z 1}$ in such a way that the controller will not act on high frequencies in the error signal by weighting the controller sensitivity by a filter with high gains at high frequencies. It is important to realize that we are trying to minimize four elements in a block using the two weights so it is important to
TABLE III

VARIANCE OF THE MFC SIGNALS FOR DIFFERENT CONTROL AND LOAD SCENARIOS. IN THE LAST TWO ROWS THE VARIANCE REDUCTION Is EXPRESSED IN A PERCENTAGE OF THE NOMINAL CASE (WITHOUT ADVANCED CONTROL)

\begin{tabular}{lll|llll} 
& & & & & \multicolumn{2}{l}{ Load reduction } \\
FB & FF & yaw & MFC blade 1 & MFC blade 2 & {$[\%]$} & {$[\%]$} \\
- & - & - & 0.149 & 0.207 & - & - \\
$\checkmark$ & - & - & 0.055 & 0.026 & 63 & 87 \\
- & $\checkmark$ & - & 0.084 & 0.134 & 44 & 36 \\
$\checkmark$ & $\checkmark$ & - & 0.014 & 0.021 & 91 & 90 \\
\hline- & - & $\checkmark$ & 0.315 & 0.300 & - & - \\
$\checkmark$ & - & $\checkmark$ & 0.073 & 0.033 & 77 & 89 \\
- & $\checkmark$ & $\checkmark$ & 0.185 & 0.143 & 42 & 53 \\
$\checkmark$ & $\checkmark$ & $\checkmark$ & 0.083 & 0.021 & 74 & 93 \\
\hline
\end{tabular}

apply appropriate scaling. The results of the synthesized feedback controller can be found in the next section.

\section{Wind TunNel Measurements}

In this section, the main results of the experiments in the wind tunnel are presented. The subspace identification experiment has already been presented in Section III. In this section, we present 3 cases, out of many, that will show the possibilities of the "smart" rotor concept using the proposed advanced control schemes. ${ }^{7}$

\section{A. Case 1: Nominal Operation With Feedback Control}

In this case we show the performance of solely the $\mathcal{H}_{\infty}$-feedback controller for nominal operation. With nominal operation we mean the wind turbine is standing perpendicular to the wind, with a wind speed of $7 \mathrm{~m} / \mathrm{s}$ and a rotational speed of $370 \mathrm{r} / \mathrm{min}$. We have a stochastic perturbations coming from the wind (turbulence) and periodic, mainly deterministic, perturbations due to the unbalance of the two-bladed rotor. In Fig. 7 we see the square-root of the smoothed power spectral density with and without the synthesized feedback controller. The data from which the figures are plotted are based on a two minute time series. ${ }^{8}$ We see, as expected, a clear performance improvement at all the periodic multiples of the rotor speed. The result is even more visible if we look at the time domain results presented in Fig. 8. In this figure we can roughly see a amplitude reduction of approximately $60 \%$. This observation is quantified in Table III where we show the variance of the signal with and without control and for different situations.

\section{B. Case 2: Nominal Operation Feedback and Feedforward Control}

In this paper we also developed an algorithm to tune our fixed-structure feedforward controller. ${ }^{9}$ In this case we show that the $\theta$ 's converge and that we obtain considerable additional

\footnotetext{
${ }^{7}$ The results are only shown for a wind speed of $7 \mathrm{~m} / \mathrm{s}$ and a rotational speed of $370 \mathrm{r} / \mathrm{min}$ since the generator broke down before we could do well-defined experiments for other conditions.

${ }^{8}$ This was too short to have well defined fatigue calculations, such as rainflow counting, which is commonly used for certification.

${ }^{9}$ The feedforward controller is tuned based on the open loop dynamics. An improvement can be made if the feedback controller and feedback controller are designed in parallel. However, in [53] they claim that a similar feedforward scheme was robust for small model variations and that was the motivation to work with one feedforward law. This to have a fair comparison between the different control layouts.
} 
$\theta$ 's on blade 1
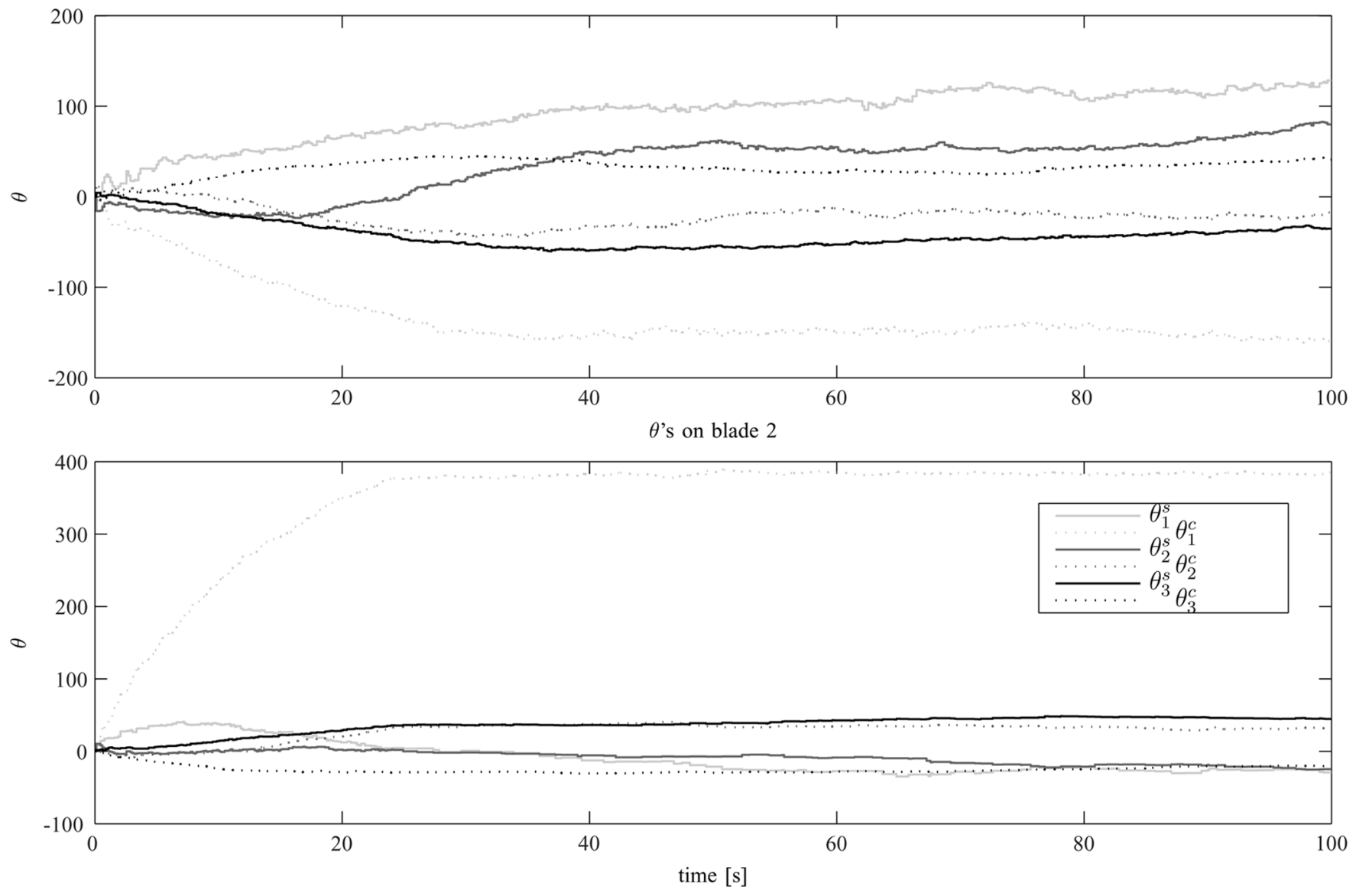

Fig. 9. Different $\theta$ 's evolving in time. In this figure we see the convergence of the feedforward scheme. For $V=7 \mathrm{~m} / \mathrm{s}$ and $\omega=370 \mathrm{r} / \mathrm{min}$ and with $\beta=0.999$ and $\alpha=0.05$.

load reduction using this feedforward scheme while also using the feedback scheme. With the feedforward scheme we will try to target the first three periodic components. With two actuators this means that we have 12 parameters that we have to estimate. This estimation is done trial wise so after every rotation. In Fig. 9 we see all these parameters plotted. The update law is derived according the formulation derived in Section IV and we used the identified model to obtain this law. So we can speak of a data-driven approach. In Fig. 9 we can see a convergence of all the $\theta$ 's. In Fig. 10 the square-root of the power-spectral density is given for the system without any control and with the combination of feedforward and feedback control. We clearly see an improvement with respect to the uncontrolled case but even more important, we see an improvement with the case where we only have feedback control (see Fig. 7). The variance of the corresponding signals, which can be seen as a rough measure for fatigue, is listed in Table III. We see that we have a load reduction of at least $90 \%$ on both sensor channels if both the feedback and the feedforward is active, which is remarkably high. The results with solely feedforward show a load reduction of approximately $40 \%$.

\section{Case 3: Yaw Misalignment}

The final case is the case where there is a misalignment between the wind direction and the axis of the rotor. This is called
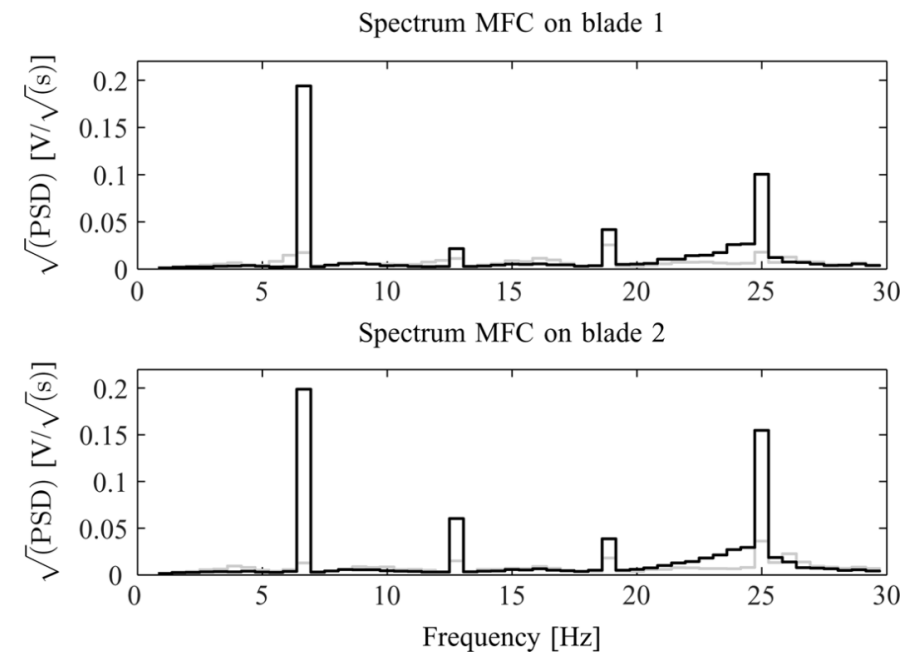

Fig. 10. Square-root of the PSD of the MFC signal on blade one and two. The grey line is with $\mathcal{H}_{\infty}$ feedback and fixed-structure feedforward control and the black one is without any control. For $V=7 \mathrm{~m} / \mathrm{s}$ and $\omega=370 \mathrm{r} / \mathrm{min}$.

yaw-misalignment, and the amount of misalignment can be expressed in degrees. For this case we have a misalignment of $5^{\circ}$. Such a misalignment will increase the load spectrum on the turbine and especially on the $1 \mathrm{P}$ frequency. This can be seen in Fig. 11 where we see an increase in the square-root of the PSD 
Spectrum MFC on blade 1

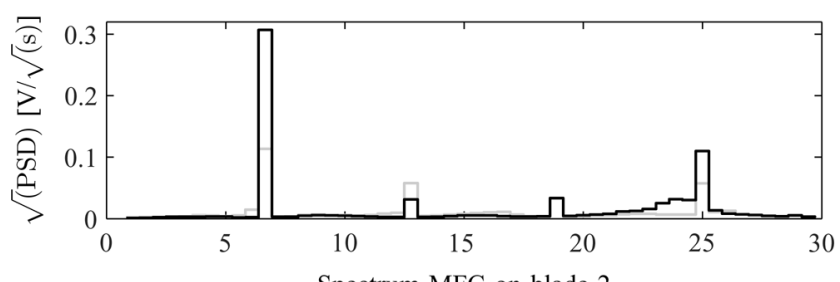

Spectrum MFC on blade 2

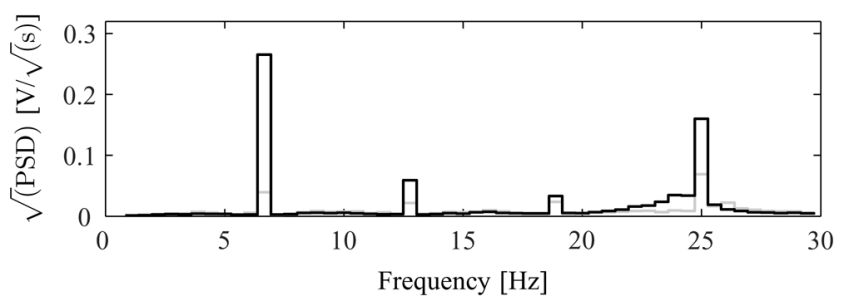

Fig. 11. Square-root of the PSD of the MFC signal on blade one and two. The grey line is with $\mathcal{H}_{\infty}$ feedback and fixed-structure feedforward control the black one is without any control. For $V=7 \mathrm{~m} / \mathrm{s}$ and $\omega=370 \mathrm{r} / \mathrm{min}$ and a yaw misalignment of $5^{\circ}$.

at the $1 \mathrm{P}$ frequency. In the same figure, we still see a significant load reduction if we use feedback and feedforward control. The results for different configurations are also included in Table III where we see comparable performance levels as the one obtained in nominal operation.

\section{CONCLUSION AND RECOMMENDATIONS}

In this paper a proof of concept study was performed that showed the feasibility of the load alleviation abilities of a rotating "smart" rotor; that is, a rotor where the blades are equipped with a number of control devices that locally change the lift profile on the blade, combined with appropriate sensors and feedback controllers. We developed dedicated identification tools to obtain experimental models to both design advanced feedback and feedforward controllers. A feedback controller based on $\mathcal{H}_{\infty}$ loop shaping was designed and we proposed to use a fixed-structure feedforward scheme. For this scheme we developed a data-driven approach to tune the design DOF which are present within this scheme. We evaluated the performance using a number of different load scenario's. We showed that with appropriate control techniques the variance of the load signals can be reduced up to $90 \%$ for both nominal operation or yaw misalignment.

Still, the "smart" rotor technology is a technology for the future and faces a number of challenges before the concept can be adopted by the industry, such as: development of suitable actuators and sensors, reliability, maintenance, integration of the actuators and sensors in a wind turbine, controller design, etc. Furthermore, the wind energy community is still to be convinced: a large number of proof of concept studies, studies in which the added value of these novel concepts is demonstrated and quantified, must be performed. We believe we contributed to this with the presented work.

\section{REFERENCES}

[1] J. Manwell, J. McGowan, and A. Rogers, Wind Energy Explained; Theory, Design and Application. New York: Wiley, 2002.
[2] E. Bossanyi, "The design of closed loop controllers for wind turbines," Wind Energy, vol. 3, no. 3, pp. 149-163, 2000.

[3] E. Bossanyi, "Individual blade pitch control for load reduction," Wind Energy, vol. 6, no. 2, pp. 119-128, 2003.

[4] E. Bossanyi, "Further load reductions with individual pitch control," Wind Energy, vol. 8, no. 4, pp. 481-485, 2005.

[5] M. H. Hansen, A. Hansen, T. Larsen, O. Stig, P. Sorensen, and P. Fuglsang, "Control design for pitch-regulated, variable-speed wind turbine," Riso Nat. Lab., Tech. Rep. Riso-R-1500(EN), 2005.

[6] K. Selvam, S. Kanev, J. W. van Wingerden, T. van Engelen, and M. Verhaegen, "Feedback-feedforward individual pitch control for wind turbine load reduction," Int. J. Robust Nonlinear Control, vol. 19, no. 1, pp. 72-91, 2009.

[7] N. D. Ham, "A simple system for helicopter individual-blade-control using modal decomposition," Vertica, vol. 4, no. 1, pp. 23-28, 1980.

[8] P. Friedmann and T. A. Millott, "Vibration reduction in rotorcraft using active control: A comparison of various approaches," J. Guid., Control, Dyn., vol. 18, no. 4, pp. 664-673, 1995.

[9] M. Lovera, P. Colaneri, C. Malpica, and R. Celi, "Closed-loop aeromechanical stability of hingeless rotor helicopters with higher harmonic control," J. Guid., Control, Dyn., vol. 29, no. 1, pp. 179-189, 2006.

[10] M. Lovera, P. Colaneri, C. Malpica, and R. Celi, "Discrete-time, closed-loop aeromechanical stability analysis of helicopters with higher harmonic control," J. Guid. Control, Dyn., vol. 30, no. 5, pp. 1249-1260, 2007.

[11] R. Barret, "Intelligent rotor blade actuation through directionally attached piezoelectric crystals," presented at the 46th Annu. Forum Technol. Display, Washington, DC, 1990.

[12] I. Chopra, "Status of application of smart structures technology to rotorcraft systems," Amer. Helicopter Soc., vol. 45, no. 4, pp. 228-252, 2000.

[13] K. Standish and C. Van Dam, "Computational analysis of a microtabbased aerodynamic load control system for rotor blades," J. Amer. Helicopter Soc., vol. 50, no. 3, pp. 249-258, 2005.

[14] B. Marrant and T. Van Holten, "Comparison of smart rotor blade concepts for large offshore wind turbines," presented at the Offshore Wind Energy Other Renewable Energies Med. Euro. Seas, Civitavecchia, Italy, 2006.

[15] T. Barlas, A. Hulskamp, J. W. van Wingerden, A. Mroz, P. van Langen, M. Landa, and S. Apinaniz, "Smart rotor blades and rotor control for wind turbines state of the art," Knowledge Base Report for UpWind WP 1B3, 2007.

[16] T. K. Barlas and G. A. M. van Kuik, "State of the art and prospectives of smart rotor control for wind turbines," J. Phys., Conf. Series: Sci. Mak. Torque From Wind, vol. 75, no. 012080, 2007.

[17] T. K. Barlas and G. A. M. van Kuik, "Review of state of the art in smart rotor control research for wind turbines," Progr. Aerosp. Sci., vol. 46, no. 1, pp. 1-27, 2010.

[18] S. Joncas, O. Bergsma, and A. Beukers, "Power regulation and optimization of offshore wind turbines through trailing edge flap control," presented at the 43th AIAA Aerosp. Sci. Meet. Exhibit (ASME), Reno, NV, 2005.

[19] T. Buhl, M. Gaunaa, and C. Bak, "Potential of load reduction using airfoils with variable trailing edge geometry," J. Solar Energy Eng., vol. 127, no. 4, pp. 503-516, 2005.

[20] S. Basualdo, "Load alleviation on wind turbine blades using variable airfoil geometry," Wind Eng., vol. 29, no. 2, pp. 169-182, 2005.

[21] M. Gaunaa, "Unsteady 2D potential-flow forces on a thin variable geometry airfoil undergoing arbitrary motion," Ris $\varnothing$, Tech. Rep. Ris $\varnothing-\mathrm{R}-$ 1478(EN), 2006.

[22] C. Bak, M. Gaunaa, P. Andersen, T. Buhl, P. Hansen, K. Clemmensen, and R. Moeller, "Wind tunnel test on wind turbine airfoil with adaptive trailing edge geometry," presented at the 45th AIAA Aerosp. Sci. Meet. Exhibit (ASME), Reno, NV, 2007.

[23] J. Zayas, C. P. V. Dam, R. Chow, J. Baker, and E. Mayda, "Active aerodynamic load control for wind turbine blades," presented at the Euro. Wind Energy Conf. (EWEC), Athens, Greece, 2006.

[24] R. Chow and C. van Dam, "Computational investigations of deploying load control microtabs on a wind turbine airfoil," presented at the 45th AIAA Aerosp. Sci. Meet. Exhibit (ASME), Reno, NV, 2007.

[25] C. P. van Dam, R. Chow, J. Zayas, and D. A. Berg, "Computational investigations of small deploying tabs and flaps for aerodynamic load control," J. Phys., Conf. Series: Sci. Mak. Torque From Wind, vol. 75, no. 012027, 2007.

[26] P. B. Andersen, M. Guanaa, C. Bak, and T. Buhl, "Load alleviation on wind turbine blades using variable airfoil geometry," presented at the Euro. Wind Energy Conf. (EWEC), Athens, Greece, 2006. 
[27] J. W. van Wingerden, A. Hulskamp, T. Barlas, B. Marrant, G. A. M. Van Kuik, D.-P. Molenaar, and M. Verhaegen, "On the proof of concept of a 'smart' wind turbine rotor blade for load alleviation," Wind Energy, vol. 11 , no. 3, pp. 265-280, 2008.

[28] D. J. Leith and W. E. Leithead, "Appropriate realization of gain-scheduled controller with application to wind turbine regulation," Int. J. Control, vol. 65, no. 2, pp. 223-248, 1996.

[29] K. A. Stol, "Dynamics modelling and periodic control of horizontalaxis wind turbines," Ph.D. dissertation, Faculty Grad. Sch., Univ. Colorado, Boulder, 2001.

[30] A. D. Wright, "Modern control design for flexible wind turbines," Ph.D. dissertation, Nat. Renewable Energy Lab., Golden, CO, 2004.

[31] M. Hand and M. Balas, "Blade load mitigation control design for a wind turbine operating in the path of vortices," Wind Energy, vol. 10, no. 4, pp. 339-355, 2007.

[32] K. Z. Østergaard, P. Brath, and J. Stoustrup, "Gain-scheduled linear quadratic control of wind turbines operating at high wind speed," presented at the 16th IEEE Int. Conf. Control Appl. (CCA), IEEE MultiConf. Syst. Control, Singapore, 2007.

[33] C. Jauch, T. Cronin, P. Sorensen, and B. B. Jensen, "A fuzzy logic pitch angle controller for power system stabilization," Wind Energy, vol. 10, no. 1 , pp. $10-39,2007$.

[34] L. C. Henriksen, "Model predictive control of a wind turbine," M.S. thesis, Informatics Math. Modell., Tech. Univ. Denmark, Denmark, 2007.

[35] L. C. Henriksen, "Model predictive control of a wind turbine with constraints," presented at the Euro. Wind Energy Conf. (EWEC), Brussels, Belgium, 2008.

[36] P. M. M. Bongers, "Modeling and identification of flexible wind turbines and a factorizational approach to robust control," Ph.D. dissertation, Mech. Eng., Delft Univ. Technol., Delft, The Netherlands, 1994.

[37] F. Bianchi, H. D. Battista, and R. Mantz, Wind Turbine Control Systems; Principles, Modelling and Gain Scheduling Design. London, U.K.: Springer-Verlag, 2007.

[38] F. Bianchi, R. Mantz, and C. Christiansen, "Control of variable-speed wind turbines by LPV gain scheduling," Wind Energy, vol. 7, no. 1, pp. $1-8,2004$.

[39] F. Bianchi, R. Mantz, and C. Christiansen, "Gain scheduling control of variable-speed wind energy conversion systems using quasi-LPV models," Control Eng. Pract., vol. 13, no. 2, pp. 247-255, 2005.

[40] F. Lescher, J. Y. Zhao, and A. Martinez, "Multiobjective $H_{2} / H_{\infty}$ control of a pitch regulated wind turbine for mechanical load reduction," presented at the Euro. Wind Energy Conf. (EWEC), Athens, Greece, 2006.

[41] K. Z. Østergaard, J. Stoustrup, and P. Brath, "Linear parameter varying control of wind turbines covering both partial and full load conditions," Int. J. Rob. Nonlinear Control, vol. 19, pp. 92-116, 2009.

[42] K. Z. Østergaard, J. Stoustrup, and P. Brath, "Rate bounded linear parameter varying control of a wind turbine in full load operation," presented at the 17th IFAC World Congr., Seoul, South-Korea, 2008.

[43] K. Z. Østergaard, "Robust, gain-scheduled control of wind turbines," Ph.D. dissertation, Autom. Control, Dept. Electron. Syst., Aalborg Univ., Aalborg, Denmark, 2008.

[44] J. W. van Wingerden, I. Houtzager, F. Felici, and M. Verhaegen, "Closed-loop identification of the time-varying dynamics of variable-speed wind turbines," Int. J. Rob. Nonlinear Control, vol. 19, no. 1, pp. 4-21, 2009.

[45] Face International Cooperation, VA, "Thunder ${ }^{\text {tm }}$ TH-6R data sheet," 2008. [Online]. Available: http://www.faceinternational.com

[46] dSPACE GmbH, Paderborn, Germany, "Website dSPACE," 2008. [Online]. Available: www.dSPACE.com

[47] Matlab, Natick, MA, "Website Matlab," 2008. [Online]. Available: http://www.mathworks.com/products/matlab

[48] Simulink, Natick, MA, "Website Simulink," 2008. [Online]. Available: http://www.mathworks.com/products/simulink

[49] M. Verhaegen, "Identification of the deterministic part of MIMO statespace models given in innovations form from input-output data," Automatica, vol. 30, no. 1, pp. 61-74, 1994.

[50] M. Verhaegen and V. Verdult, Filtering and System Identification: A Least Squares Approach. Cambridge, U.K.: Cambridge Univ. Press, 2007.

[51] A. Chiuso, "The role of vector auto regressive modeling in predictor based subspace identification," Automatica, vol. 43, no. 6, pp. 1034-1048, 2007.
[52] G. H. Golub and C. F. v. Loan, Matrix Computations. Baltimore, MD: The John Hopkins University Press, 1996.

[53] S. H. van de Meulen, R. L. Tousain, and O. H. Bosgra, "Fixed structure feedforward controller design exploiting iterative trials: Application to a wafer stage and a desktop printer.," J. Dyn. Syst., Meas. Control, vol. 130 , no. 5, 2008 .

[54] H. Balini, I. Houtzager, J. Witte, and C. W. Scherer, "Subspace identification and robust control of an AMB system," in Amer. Control Conf., 2010.

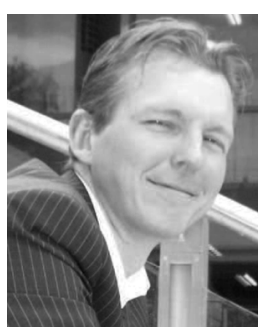

Jan-Willem van Wingerden was born on December 9, 1980 in Ridderkerk, The Netherlands. He received the B.S. degree cum laude and the Ph.D. degree Cum Laude from the Delft Center of Systems and Control, the Delft University of Technology, Delft, The Netherlands, in 2004 and 2008, respectively. His Ph.D. project was entitled 'Smart' dynamic rotor control for large offshore wind turbines.

His graduation project was carried out at Philips Applied Technologies in Eindhoven. Currently he is an Assistant Professor with the Delft University of Technology. His main research interests include LPV identification, subspace identification, 'Smart' structures, and control and identification of wind turbines.

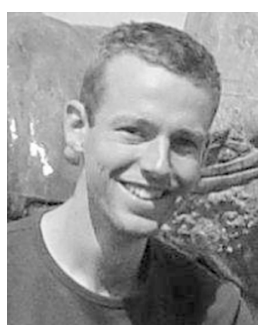

Anton Hulskamp was born in Amsterdam, The Netherlands, in April, 1980. He received the B.S. degree in mechanical engineering from the University of Twente, Twente, The Netherlands. He is currently pursuing the Ph.D. degree in the structural aspects of smart rotor blades for wind turbines from the Faculty of Aerospace Engineering, Delft University of Technology, Delft, The Netherlands, where his focus is on on adaptive materials and structural integration.

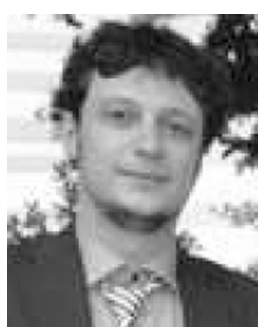

Thanasis Barlas received the Diploma and M.Sc. degree, both in mechanical engineering, from University of Thessaly, Volos, Greece, with a specialization in advanced energy systems and did his thesis project on analysis of aeroelastic loads of wind turbines in unsteady inflow. He is currently pursuing the Ph.D. degree from the Delft University Wind Energy Research Institute, Delft University of Technology, Delft, The Netherlands.

$\mathrm{He}$ has been working in wind energy research for the last 5 years and is currently involved in the E.U.'s Upwind Project, investigating smart rotor blades and rotor control. His research is focusing on aeroelastic modeling and control of wind turbines. His research interests include aeroelasticity, unsteady aerodynamics, near wake aerodynamics and controls.

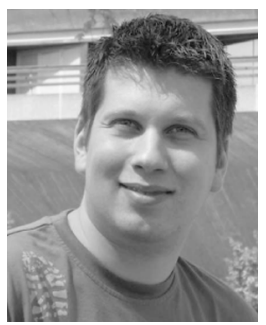

Ivo Houtzager received the B.Eng. degree in mechanical engineering from Rotterdam University of Applied Sciences, Rotterdam, The Netherlands, in 2003 and the M.Sc. degree in systems and control engineering from Delft University of Technology, Delft, The Netherlands, in 2007, where he is currently pursuing the Ph.D. degree in control engineering.

His research interest is the development of identification methods and inversion methods for large-scale dynamical systems and the use of the identification and inversion results in tuning controllers, as well as towards applications of the developed methods and techniques in the light weight structures and the wind energy. 


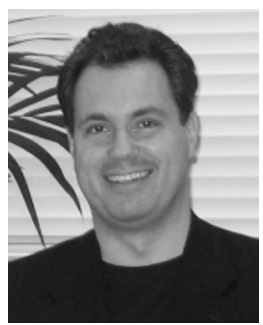

Harald Bersee is a full time Associate Professor with the Research Group DPCS with a specialization on composite materials and processes. Previous relevant experience includes setting up a research line on composites materials and processes with an emphasis on thermoplastic composites. Four years ago, he started up the research into the development of resin infusion of thermoplastic composites, resulting in 1 patent on the modified resin and numerous publications. The research area has been extended with the development of smart structures for both aerospace and wind energy. Currently there is a strong involvement in the JTI Smart Fixed Wing Aircraft (SFWA) Project through the position as the level 2 leader of the system integration work package.

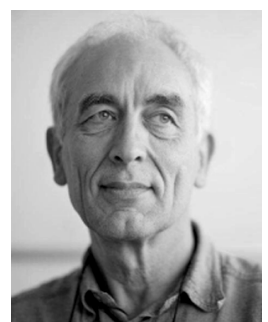

Gijs van Kuik received the B.S. degree from the Faculty of Aerospace Engineering, Delft University of Technology, Delft, The Netherlands, in 1977, with specialization on theoretical aerodynamics, and the $\mathrm{Ph} . \mathrm{D}$. degree in theoretical aspects of wind turbine aerodynamics from Eindhoven University of Technology, Eindhoven, The Netherlands, in 1988.

Hereafter he became one of the first researchers in the Delft University wind energy research, focusing on the aerodynamics and dynamics of the rotor. From 1988, he was employed by Stork Product
Engineering, involved in technology development and design of wind turbines for all Dutch and some European manufacturers. In 1998, he returned to Delft University to become a Professor in Wind Energy. From 2000, he is the Scientific Director of the Delft University Wind Energy Research Institute DUWIND. He is the initiator of the research program on "Smart Wind Turbine Rotors" or rotors with distributed control capabilities, and coordinates a European Research Project on this topic.

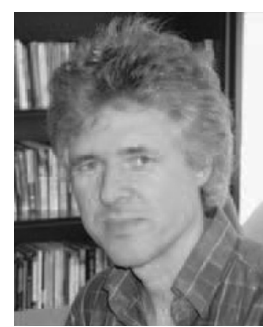

Michel Verhaegen received the engineering degree in aeronautics from the Delft University of Technology, Delft, The Netherlands, in 1982, and the doctoral degree in applied sciences from the Catholic University, Leuven, Belgium, in 1985.

From 1985 to 1994, he has been a Research Fellow of the U.S. National Research Council (NRC) and the Dutch Academy of Arts and Sciences. In the period 1994-1999 he was an Associate Professor of the Control Laboratory of the Delft University of Technology and became a full Professor at the faculty of Applied Physics of the University of Twente, Twente, The Netherlands, in 1999. From 2001 on, he moved back to the University of Delft and joined the Delft Center for Systems and Control. His main research directions include system identification, distributed and fault tolerant control, and data driven controller design methodologies. Application areas include smart structures, swarms of satellites, adaptive optics, wind energy, and vehicle mechatronics. 